\title{
Common Elements in Semantic Descriptions of Web Processing Services
}

\author{
Barbara Hofer \\ Interfaculty Department of Geoinformatics - Z_GIS, University of Salzburg, Austria · \\ barbara.hofer@sbg.ac.at
}

Short paper

\begin{abstract}
Web Processing Services (WPS) offer geoprocessing functionalities online. The description of an offered process to date consists of a textual description of the operation, and a specification of input and output parameters. This description is limited in detail and makes search, discovery, and composition of services difficult. Two approaches have been proposed to extend these service descriptions: ontology-based descriptions and WPS profiles, with differing complexity and intended use. This contribution confronts the two approaches, and suggests structured metadata as a linkage between service profiles and ontologies as future work.
\end{abstract}

\section{Introduction}

The suite of web service interface specifications of the Open Geospatial Consortium (OGC) is meant to facilitate resource integration and interoperability. Data providing services such as web maps, feature, and coverage services are well established, whereas only a small fraction of web services provide geoprocessing functionalities (LOPEZ-PELLICER et al. 2012). Nevertheless, a series of examples demonstrates the use of web processing services (WPS), the OGC standard for providing online geoprocessing. WPS have been used in theme-specific platforms like a platform for analysing the spread of diseases (DOMINKOVICS et al. 2011), to provide specific processing functionalities like coordinate transformations (SUN \& YUE 2010), to expose geoprocessing procedures (termed models) as services (CANNAT et al. 2012), etc. The objectives of using online geoprocessing technology on a general level are to increase interoperability and to support collaboration (HOFER 2014).

The generic nature of process descriptions is one limiting factor for the wide application of WPS (JONES et al. 2012). The WPS interface specification defines how to interact with a service on a syntactic level. Available requests are GetCapabilities, DescribeProcess and Execute, whereby the responses of GetCapabilities and DescribeProcess contain information on parameter values required for the Execute request. In addition to the syntactic specifications, information on a semantic level is required for a successful interoperation of these services. BRAUNER et al. (2009) identified semantic descriptions as an element of a resaerch agenda for online geoprocessing; the other areas listed in the agenda are orchestration, which requires semantic descriptions as well, and improvement of performance (BRAUNER et al. 2009).

GI_Forum - Journal for Geographic Information Science, 1-2015.

(C) Herbert Wichmann Verlag, VDE VERLAG GMBH, Berlin/Offenbach. ISBN 978-3-87907-558-4.

(C) ÖAW Verlag, Wien. ISSN 2308-1708, doi:10.1553/giscience2015s2. 
Semantic annotations and deep service descriptions of web services have been subject of various works (LEMMENS et al. 2006, LUTZ 2007, FITZNER et al. 2011). These approaches are all using ontologies or thesauri for linking a term used in the service description and its documented meaning. An alternative approach to describing the meaning of an operation and its inputs and outputs in more detail are WPS profiles (MÜLLER 2013). These profiles have been foreseen since the WPS 1.0 specification, but were not used much. In the WPS 2.0 interface standard, which has been recently published, WPS profiles are specified in more detail (MÜLLER \& PROSS 2015).

Although the anticipated uses of ontology-based and profile-based descriptions of WPS seem different (section 2), they are both encoding key elements for service descriptions. A comparison of the two approaches highlights these key elements (section 3) and constitutes early stage research results. The comparison is also the basis for a future investigation of a potential mediation between the two approaches, which would leverage the advantages of both approaches. The mediation could take form of structured metadata for describing processing services in a semi-formal way as proposed in (ANSELIN 2012, MÜLLER 2013).

\section{Approaches to Adding Semantics to WPS}

The difficulty that needs to be addressed with semantic descriptions of WPS is that a mere description of input and output is not sufficient. The description needs to specify the relationship between input and output as well (LUTZ 2007). Syntactic signatures, which contain only input and output parameters of an operation, can be identical for different operations or different for similar functionality (LUTZ 2007). As an example, the operations intersection and union, both have the same input and output (FITZNER et al. 2011). A search operation that focuses on syntactic signatures only, cannot differentiate between operations with identical signatures.

One of the approaches mentioned above is the approach by (LUTZ 2007). He works with the specification of semantically annotated syntactic signatures and the definition of pre- and postconditions for a high level of detail in service descriptions. For example, in case of an intersection operation, that would mean to additionally state that the two input vectors need to be in the same coordinate reference system. His approach encodes the relationship between input and output and allows a differentiation between operations on a higher level of detail in automated reasoning. The approach has the disadvantage that two separate formalizations need to be employed in ontologies and that the reasoning with pre- and postconditions is not always decidable.

An alternative to ontology-based approaches has been proposed for the WPS 2.0 specification: WPS profiles. A WPS profile provides a documentation of an operation similar to an entry in a help document in a piece of software. MÜLLER (2013) introduced a hierarchical framework of WPS profiles, which provides the basis for the WPS 2.0 specification (MÜLLER \& PROSS 2015). Three profiles are suggested: (1) a process concept profile, (2) a process generic profile, and (3) a process implementation profile. The concept profile contains a description of the general functioning of the operation and a unique identifier. The generic profile specifies an abstract interface for a process including abstract data types for input and output and parameter names. This profile is extended by an implementation 
profile that mentions specific data formats. These profiles will be available as HTML documents that are linked to service descriptions as entries to metadata fields.

Since the profiles are not fully machine-interpretable, they seem to serve a specification of the semantics of operations for human users. The WPS profile approach is less complex than an ontology-based approach, because it does not require the availability of ontologies and related reasoning capabilities.

\section{Elements of Semantic Descriptions}

The hypothesis of this research is that the structure suggested in process ontologies as described above, can serve as a structure for service metadata contained in WPS profiles. The required elements for describing processes in ontologies are covered in pre- and postconditions of the operations, together with shared variables. This information is contained in textual descriptions of spatial operations as well. A structure for separating these elements may facilitate the process of filling WPS profiles, and of deriving ontologies in the long run. Lists of abstract data types or controlled vocabularies of conditions may further support the completion of profiles. At the same time, a structured approach to filling WPS profiles may facilitate the translation of profiles into operation ontologies in the future.

The following example illustrates the description of a buffer operation in a WPS generic profile provided as text in (MÜLLER \& PROSS 2015) with the proposed metadata structure, which is based on elements of process ontologies:

Precondition: geometric object with name and spatial reference system; distance parameter with name and data type.

Postcondition: geometric object with name and the same spatial reference system as the input geometric object, containing points around the geometric input object less than or equal to the specified distance.

SharedVariables: input geometric object, distance parameter, spatial reference system.

This structured description of an operation follows the elements proposed in web service ontologies. It adheres to the pragmatics of WPS profiles, but may eventually allow a linkage to operation ontologies. These ontologies are considered important as semantic web technologies support automation of discovery and composition of services.

Certainly, the description in WPS profiles will remain less formal than an entry in an ontology. The creation of controlled vocabularies for filling the profiles as proposed in (MÜLLER 2013) may increase formality of the description. These first observations suggest a further evaluation of the level of detail of operation specification in ontology-based approaches, as well as the development of tools and controlled vocabularies to fill WPS profiles considering the identified elements of structured descriptions. 


\section{Acknowledgments}

Thanks to the reviewers of this contribution for their comments. This work was supported by a DRESDEN junior fellowship of the Technical University of Dresden, Germany.

\section{References}

Anselin, L. (2012), From SpaceStat to CyberGIS: Twenty Years of Spatial Data Analysis Software. International Regional Science Review, 35 (2), 131-157.

Brauner, J., Foerster, T., Schaeffer, B. \& BArAnski, B. (2009), Towards a Research Agenda for Geoprocessing Services. 12th AGILE International Conference on Geographic Information Science. J. Haunert, B. Kieler \& J. Milde. Leibniz University Hannover, Germany.

Cannata, M., Molinari, M. E., Xuan Luan, T. \& Hung Long, N. (2012), Web Processing Services for shallow landslide. International Journal of Geoinformatics, $8(1), 25-34$.

Dominkovics, P., Granell, C., Pérez-Navarro, A., Casals, M., Orcau, À., \& Caylà, J. A. (2011), Development of spatial density maps based on geoprocessing web services: application to tuberculosis incidence in Barcelona, Spain. International Journal of Health Geographics, 10.

FitzNer, D., HoffmanN, J. \& KLIEN, E. (2011), Functional description of geoprocessing services as conjunctive datalog queries. Geoinformatica, 15 (1), 191-221.

HOFER, B. (2014), Uses of online geoprocessing technology in analyses and case studies: a systematic analysis of literature. International Journal of Digital Earth, 1-17 (published online: http://www.tandfonline.com/doi/abs/10.1080/17538947.2014.962632).

Jones, R., Cornford, D. \& BAstin, L. (2012), UncertWeb Processing Service: Making Models Easier to Access on the Web. Transactions in GIS, 16 (6), 921-939.

Lemmens, R., Granell, C., Wytzisk, A., De By, R., Gould, M. \& Van Oosterom, P. (2006), Integrating Semantic and Syntactic Descriptions to Chain Geographic Services. Internet Computing, IEEE, 10 (5), 42-52.

Lopez-Pellicer, F. J., Rentería-Agualimpia, W., BéJAR, R., Muro-Medrano, P. R. \& ZARAZAGA-SORIA, F. J. (2012), Availability of the OGC geoprocessing standard: March 2011 reality check. Computers \& Geoscience, 47 (0), 13-19.

LuTZ, M. (2007), Ontology-Based descriptions for semantic discovery and composition of geoprocessing services. Geoinformatica, 11 (1), 1-36.

MÜLLER, M. (2013), Hierarchical process profiles for interoperable geoprocessing functions. Proceedings of the 16th AGILE Conference on Geographic Information Science, Leuven, Belgium.

MÜller, M. \& PROSS, B. (2015), OGC WPS 2.0 Interface Standard, OGC. http://docs.opengeospatial.org/is/14-065/14-065.html.

OGC (2012), Semantic Annotations in OGC Standards. OGC Best Practices, Open Geospatial Consortium, 59.

Sun, Z. \& YuE, P. (2010), The use of Web 2.0 and geoprocessing services to support geoscientific workflows. 